\title{
Identifying Myeloma Patients at Risk of Lenalidomide or Pomalidomide Resistance at Relapse: A New Opportunity
}

\author{
Suvir Singh ${ }^{1, \odot}$ \\ 1Department of Clinical Hematology and Stem Cell Transplantation, \\ Dayanand Medical College, Ludhiana, Punjab, India
}

Ind J Med Paediatr Oncol 2021;42:180-181.

Gooding et al reported an interesting study that demonstrated the presence of acquired genetic changes in cereblon in patients with multiple myeloma refractory to lenalidomide or pomalidomide. ${ }^{1}$ We provide a short summary and appraisal of this study and implications for clinical practice.

\section{Background}

Cereblon (CRBN) is a crucial component of the E3 ubiquitin ligase complex and marks substrate proteins for proteolysis. Binding of lenalidomide (Len) or pomalidomide (Pom) to CRBN leads to proteolysis of Ikaros family zinc finger 1 (IKZF1) and IKZF3, leading to myeloma cell death. ${ }^{2}$

\section{Brief Summary of This Study}

This was an in-vitro study, in which DNA and RNA extracted from CD138 enriched plasma cells and peripheral blood germline DNA were collected at baseline and relapse in patients with multiple myeloma (MM). These cells were analyzed using whole genome sequencing and RNA sequencing to obtain germline and tumor data with a focus on CRBN. Significant findings included the definition of several mutations in CRBN, broadly classified as point mutations, copy number losses, and a distinct exon-10-deleted variant. Overall frequency of mutations was noted to progressively increase with subsequent lines of treatment, being $0.9 \%$ in newly diagnosed multiple myeloma to $2.2 \%$ in Len refractory and $9 \%$ in Pom refractory patients. Strikingly, structural variation or loss of CRBN expression was noted in $8 \%$ of Len refractory and $24 \%$ of Pom refractory patients. A novel exon-10-deleted CRBN transcript was defined, which leads to deletion of the Len/Pom binding region on CRBN. Frequency of exon-10-deleted CRBN was noted in $20 \%$ of Len refractory and $29 \%$ of Pom refractory patients. Patients who were Len refractory and had CRBN alterations had a significant reduction in progression free and
Address for correspondence Suvir Singh, MD, Department of Clinical Haematology, Haemato-Oncology and Bone Marrow (Stem Cell) Transplantation, Dayanand Medical College, Ludhiana, Punjab, India (e-mail: suvirs@gmail.com).

overall survival compared with those without any changes. This is the first study that illustrates acquired mutations in target proteins leading to immunomodulatory drug (IMiD) resistance, independent of CRBN gene expression.

\section{Relevance}

The current study has significant implications for routine treatment of MM and represents another step in individualizing therapy at the time of relapse. The central role played by CRBN in mediating IMiD activity previously led to conjecture that quantitative or qualitative CRBN alterations can mediate responses to therapy with Len/Pom. Patients who have a high expression of CRBN demonstrate better responses to Len. ${ }^{3}$ Conversely, patients with very low expression of CRBN show no response to a Len/Dex combination. ${ }^{4}$ The weight of evidence so far points at a quantitative reduction in the expression of CRBN as the crucial step in mediating drug resistance. ${ }^{5}$ Other qualitative changes in CRBN, that is, mutations in CRBN pathway and downstream proteins have been found to be infrequent, even in patients with clinical resistance to Len/Pom. ${ }^{6}$ It was suggested that CRBN function may be altered with other mechanisms including epigenetic and posttranscriptional changes. ${ }^{7}$ Despite this data, a role of CRBN expression in identifying patients at risk of clinical resistance has not been proven. ${ }^{8,9}$

The present study impacts both the above paradigms and has implications for the near future. The prognostic impact of mutations in this study is independent of CRBN gene expression, and may annul the results of previous studies that largely relied on quantitative CRBN expression. Myeloma is associated with a nearly $100 \%$ risk of relapse, and selection of appropriate therapy at relapse improves progression free and overall survival. ${ }^{10}$ This study provides a potential mechanism for utilizing CRBN mutations as predictors of IMiD responsiveness. As mutations in CRBN occur more frequently
DOI https://doi.org/ 10.1055/s-0041-1730090 ISSN 0971-5851 (c) 2021. Indian Society of Medical and Paediatric Oncology

This is an open access article published by Thieme under the terms of the Creative Commons Attribution-NonDerivative-NonCommercial-License, permitting copying and reproduction so long as the original work is given appropriate credit. Contents may not be used for commercial purposes, or adapted, remixed, transformed or built upon. (https://creativecommons.org/licenses/by-nc-nd/4.0/).

Thieme Medical and Scientific Publishers Private Ltd. A-12, Second Floor, Sector -2, NOIDA -201301, India 
at disease progression, a clinical algorithm may be devised, with a plan to document $C R B N$ mutations at the first instance of progression in Len/Pom exposed patients. Identification of mutations that preclude a response will allow selection of alternate effective agents. Treatment of myeloma is already set to become "personalized" based on baseline genetic profile, and this study provides a guidepost at the time of first relapse. ${ }^{11}$

There appear to be two major hurdles before this clinical model can be adopted for regular use. First, a large multicenter database of commonly occurring CRBN mutations and their association with drug resistance and clinical outcomes must be defined, and only those predicting for high rates of drug resistance (80-90\%) must influence a change in therapy. Second, the best method for examining CRBN mutations is still contentious. Most studies have used DNA and RNA extracted from CD38+ plasma cells for the evaluation of CRBN mutations, but several variations exist. CRBN mutations can be tested by targeted polymerase chain reaction, gene sequencing, or next-generation sequencing techniques. The present study used whole genome sequencing, which may not be universally available. Several methodological variables like the source of MM cells, method of purification, and identification of altered splice variants of CRBN must be harmonized so that a common protocol can be used. ${ }^{8}$

Identification of mutations in CRBN is a great first step in identification of patients at risk of resistance to Len/Pom at relapse. Future studies would need to evaluate the association of CRBN modifications with geographical variations and disease presentation. A model similar to tyrosine kinase mutations in chronic myeloid leukemia at disease progression can be envisioned and may prove to be a first step in personalized medicine at relapse in myeloma.

\section{Conflict of Interest}

None declared.

\section{References}

1 Gooding S, Ansari-Pour N, Towfic F, et al. Multiple cereblon genetic changes are associated with acquired resistance to lenalidomide or pomalidomide in multiple myeloma. Blood 2021;137(2):232-237

2 Krönke J, Udeshi ND, Narla A, et al. Lenalidomide causes selective degradation of IKZF1 and IKZF3 in multiple myeloma cells. Science 2014;343(6168):301-305

3 Klimowicz A, Neri P, Belch A, et al. High cereblon protein expression correlates with improved response and survival in myeloma patients treated with lenalidomide. American Society of Hematology Blood 2012;120(21):931

4 Schuster SR, Kortuem KM, Zhu YX, et al. The clinical significance of cereblon expression in multiple myeloma. Leuk Res 2014;38(1):23-28

5 Franssen LE, Nijhof IS, Couto S, et al. Cereblon loss and up-regulation of c-Myc are associated with lenalidomide resistance in multiple myeloma patients. Haematologica 2018;103(8):e368-e371

6 Dimopoulos K, Fibiger Munch-Petersen H, Winther Eskelund C, et al. Expression of CRBN, IKZF1, and IKZF3 does not predict lenalidomide sensitivity and mutations in the cereblon pathway are infrequent in multiple myeloma. Leuk Lymphoma 2019;60(1):180-188

7 Thakurta A, Gandhi AK, Waldman MF, et al. Absence of mutations in cereblon (CRBN) and DNA damage-binding protein 1 (DDB1) genes and significance for IMiD therapy. Leukemia 2014;28(5):1129-1131

8 Lodé L, Amiot M, Maiga S, et al. Cereblon expression in multiple myeloma: not ready for prime time. $\mathrm{Br} \mathrm{J}$ Haematol 2013;163(2):282-284

9 Qian X, Dimopoulos MA, Amatangelo M, et al. Cereblon gene expression and correlation with clinical outcomes in patients with relapsed/refractory multiple myeloma treated with pomalidomide: an analysis of STRATUS. Leuk Lymphoma 2019;60(2):462-470

10 Rajkumar SV, Kumar S. Multiple myeloma current treatment algorithms. Blood Cancer J 2020;10(9):94

11 Pawlyn C, Davies FE. Toward personalized treatment in multiple myeloma based on molecular characteristics. Blood 2019;133(7):660-675 